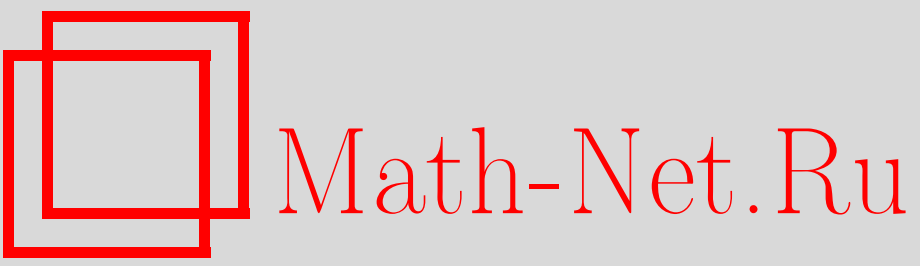

Ф. М. Мухамедов, У. А. Розиков, Об алгебре фон Неймана, соответствующей одной фазе неоднородной модели Поттса на дереве Кэли, ТМФ, 2001, том 126 , номер 2, 206-213

DOI: https://doi.org/10.4213/tmf425

Использование Общероссийского математического портала Math-Net.Ru подразумевает, что вы прочитали и согласны с пользовательским соглашением

http://www. mathnet.ru/rus/agreement

Параметры загрузки :

IP : 54.210 .77 .194

26 апреля 2023 г., 15:57:42 
ТЕОРЕТИЧЕСКАЯ

И МАТЕМАТИЧЕСКАЯ

ФИЗИКА

Том 126, № 2

февраль, 2001

(C) 2001 г.

Ф. М. Мухамедов*, У. А. Розиков*

\section{ОБ АЛГЕБРЕ ФОН НЕЙМАНА, СООТВЕТСТВУЮЩЕЙ ОДНОЙ ФАЗЕ НЕОДНОРОДНОЙ МОДЕЛИ ПОТТСА НА ДЕРЕВЕ КЭЛИ}

Доказано, что алгебра фон Неймана, порожденная при ГНС-представлении, ассоциированном с экстремальной неупорядоченной фазой одного класса неоднородных моделей Поттса на дереве Кэли, является фактором типа III $_{1}$.

\section{1. ВВЕДЕНИЕ}

Большинство проблем теории многих тел, представляющих физический интерес, довольно сложны и порой неразрешимы. Поэтому сушественный интерес приобретают модельные системы, допускаюшие их математическое рассмотрение. Одной из таких моделей является неоднородная модель Поттса.

Дерево Кэли $\Gamma^{k}=(V, L)$ порядка $k \geqslant 1$ есть бесконечное дерево, т.е. графб без циклов, из каждой вершины которого выходит ровно $k+1$ ребер, где $V$ - множество вершин $\Gamma^{k}$ и $L$ - множество его ребер.

Рассмотрим неоднородную модель Поттса, гамильтониан которой имеет вид

$$
H(\sigma)=-\sum_{\langle x, y\rangle \in L} J_{x y} \delta_{\sigma(x) \sigma(y)},
$$

где $J_{x, y} \in \mathbb{R}, \sigma(x) \in \Phi=\left\{\sigma_{1}, \sigma_{2}, \ldots, \sigma_{q}\right\} \subset \mathbb{R}^{q-1}$,

$$
\sigma_{i} \sigma_{j}= \begin{cases}1, & \text { если } i=j, \\ -\frac{1}{q-1}, & \text { если } i \neq j\end{cases}
$$

и $\delta$ - символ Кронекера.

Одним из центральных вопросов является определение типа алгебры фон Неймана, порождаемой при ГНС-представлении, ассоциированном с гиббсовскими состояниями (см. [1], [2]). Например, Пауэрс в работе [3] рассматривал свободную решетчатую квантовую спиновую систему с двумя состояниями в каждой точке, причем первое состояние

${ }^{*}$ Институт математики им. В. И. Романовского АН РУз, Ташкент, Республика Узбекистан 
занято с вероятностью $p$, а второе - с вероятностью $q=1-p$, и тогда соответствующая алгебра фон Неймана является фактором типа $\mathrm{III}_{\lambda}$, где $\lambda=p / q$.

В [4] рассматривалась модель Изинга на решетке Бете (дереве Кэли) порядка $k$ $(k \geqslant 2)$. Для экстремальных трансляционно-инвариантных гиббсовских состояний этой модели найдены связи между алгебрами фон Неймана, порожденными при ГНС-представлении этими состояниями, и показано, что эти алгебры могут иметь типы $\mathrm{III}_{\lambda}$ и $\mathrm{III}_{1}$.

Эта работа является продолжением работы [5], в которой было найдено достаточное условие экстремальности неупорядоченной фазы неоднородной модели Поттса. В настоящей работе определяется тип алгебры фон Неймана, соответствующей экстремальной неупорядоченной фазе одного класса неоднородных моделей Поттса.

\section{2. ОПРЕДЕЛЕНИЯ И ПРЕДВАРИТЕЛЬНЫЕ РЕЗУЛЬТАТЫ}

Для полноты изложения приведем некоторые определения и необходимые результаты.

Из (2) следует, что для любых $x, y \in V$

$$
\delta_{\sigma(x) \sigma(y)}=\frac{q-1}{q}\left(\sigma(x) \sigma(y)+\frac{1}{q-1}\right) .
$$

Следовательно, $H(\sigma)$ имеет вид

$$
H(\sigma)=-\sum_{\langle x, y\rangle \in L} J_{x y}^{\prime} \sigma(x) \sigma(y)
$$

где

$$
J_{x y}^{\prime}=\frac{q-1}{q} J_{x y} .
$$

Введем обозначения:

$$
W_{n}=\left\{x \in V \mid d\left(x, x^{0}\right)=n\right\},
$$

где $x^{0} \in V$ - фиксированная точка, а $d(x, y)$ - число ребер кратчайшего пути, соединяющего $x$ и $y$;

$$
\begin{gathered}
V_{n}=\bigcup_{m=1}^{n} W_{m}=\left\{x \in V \mid d\left(x, x^{0}\right) \leqslant n\right\} ; \\
L_{n}=\left\{l=\langle x, y\rangle \in L \mid x, y \in V_{n}\right\} ; \\
\theta_{x y}=\exp \left(\frac{J_{x y}^{\prime}}{T}\right) ; \\
a(x)=\sum_{y \in S(x)}\left[(q-1) \frac{\theta_{x y}-1}{\theta_{x y}}\right]^{2}, \quad x \in V ; \\
b(x)=\sum_{y \in S(x)}\left|J_{x y}^{\prime}\right|, \quad x \in V .
\end{gathered}
$$


Пусть $a(x)$ удовлетворяет одному из следуюших условий.

Условие 1: а) $a(x) \equiv 1$ для любого $x \in V$;

б) для любого $x \in V$ и любых бесконечных путей $\left\{x, x_{1}, x_{2}, \ldots\right\}$ выполняется равенство

$$
\prod_{i=1}^{\infty} a\left(x_{i}\right)=0
$$

Пусть $b(x)$ удовлетворяет следуюшему условию.

Условие 2. Сушествует $M>0$ такое, что $b(x) \leqslant M$ для любого $x \in V$.

Основным результатом работы [5] была следуюшая теорема.

Теорема 1. Если выполняются условия 1 и 2, то неупорядоченная фаза неоднородной модели Поттса на дереве Кәли является әкстремальной.

Напомним некоторые факты из теории алгебр фон Неймана. Пусть $B(H)$ - алгебра всех ограниченных линейных операторов на гильбертовом пространстве $H$ (над полем комплексных чисел).

Слабо (операторная) замкнутая *-подалгебра $\mathcal{N}$ в $B(H)$ называется алгеброй фон Неймана, если она содержит тождественный оператор $\mathbb{1}$. Алгебру фон Неймана называют фактором, если ее центр $Z(\mathcal{N})(=\{x \in \mathcal{N}: x y=y x \forall y \in \mathcal{N}\})$ тривиален, т.е.

$$
Z(\mathcal{N})=\{\lambda \mathbb{1} \mid \lambda \in \mathbb{C}\}
$$

Элемент $x \in \mathcal{N}$ называется положительныцм, если сушествует элемент $y \in \mathcal{N}$ такой, что $x=y^{*} y$. Совокупность всех положительных элементов $\mathcal{N}$ обозначается $\mathcal{N}_{+}$. Линейный функционал $\omega$ на $\mathcal{N}$ называется состоянием, если $\omega(x) \geqslant 0$ для всех $x \in \mathcal{N}_{+}$ и $\omega(\mathbb{1})=1$. Состояние $\omega$ называется нормальныцм, если для любой ограниченной возрастаюшей сети $\left\{x_{\alpha}\right\}$ положительных элементов из $\mathcal{N}$ выполнено

$$
\omega\left(\sup _{\alpha} x_{\alpha}\right)=\sup _{\alpha} \omega\left(x_{\alpha}\right) .
$$

Состояние $\omega$ называется точныц, если из $\omega\left(x^{*} x\right)=0$ следует $x=0$.

Пусть $\mathcal{N}$ - фактор, $\omega$ - точное нормальное состояние на $\mathcal{N}$ и $\sigma_{t}^{\omega}$ - модулярная группа, ассоциированная с $\omega$ на $\mathcal{N}$ (см. определение 2.5 .15 в [6]). Через $\Gamma\left(\sigma^{\omega}\right)$ обозначим спектр Конна модулярной группы $\sigma_{t}^{\omega}$ (см. определение 2.2 .1 в [7]).

ОПРЕДЕЛЕНИЕ [7]. Фактор $\mathcal{N}$ имеет тип:

$\mathrm{III}_{1}$, если $\Gamma\left(\sigma^{\omega}\right)=\mathbb{R}$;

$\mathrm{III}_{\lambda}$, если $\Gamma\left(\sigma^{\omega}\right)=\{n \ln \lambda, n \in \mathbb{Z}\}, \lambda \in(0,1)$;

$\mathrm{III}_{0}$, если $\Gamma\left(\sigma^{\omega}\right)=0$.

Более подробные сведения об алгебрах фон Неймана и модулярной теории операторных алгебр можно найти, например, в [6], [8]. 


\section{3. ОСНОВНЫЕ РЕЗУЛЬТАТЫ}

В этом разделе мы рассмотрим неоднородную модель Поттса на дереве Кэли $\Gamma^{k}$, т.е.

$$
H(\sigma)=-\sum_{\langle x, y\rangle \in L} J_{x y} \delta_{\sigma(x) \sigma(y)}
$$

где

$$
J_{x y}=\left\{\begin{array}{ll}
J, \quad \text { если } x \in W_{2 n}, \quad y \in W_{2 n+1}, \quad n \geqslant 0, \\
-J, \quad \text { если } x \in W_{2 n-1}, \quad y \in W_{2 n}, \quad n \geqslant 1,
\end{array} \quad\langle x, y\rangle \in L, \quad J>0 .\right.
$$

Через $\mu_{0}$ обозначим неупорядоченную фазу рассматриваемой модели. Непосредственно проверяя условия теоремы 1 , находим, что при

$$
\frac{(q-1) k^{\frac{1}{2}}-1}{(q-1) k^{\frac{1}{2}}}<\theta<\frac{(q-1) k^{\frac{1}{2}}}{(q-1) k^{\frac{1}{2}}-1},
$$

где

$$
\theta=\exp \left\{\frac{q J}{(q-1) T}\right\}
$$

неупорядоченная фаза $\mu_{0}$ является экстремальной. Далее мы будем предполагать, что условие (5) выполняется.

Рассмотрим $C^{*}$-алгебру

$$
A=\bigotimes_{\Gamma^{k}} M_{2}(\mathbb{C})
$$

где $M_{2}(\mathbb{C})$ - алгебра матриц $2 \times 2$ над полем комплексных чисел $\mathbb{C}$. Через $e_{i j}, i, j=1,2$, обозначим матричные единишы алгебры $M_{2}(\mathbb{C})$. Коммутативную подалгебру алгебры $M_{2}(\mathbb{C})$, порождаемую элементами $e_{11}$ и $e_{22}$, обозначим через $\mathrm{CM}_{2}(\mathbb{C})$. Положим $\mathrm{C} A=$ $\bigotimes_{\Gamma^{k}} \mathrm{C} M_{2}(\mathbb{C})$. Элементы коммутативной алгебры $\mathrm{C} A$ будем представлять как функции на пространстве $\Omega=\left\{e_{11}, e_{22}\right\}^{\Gamma^{k}}$. Пусть на измеримом пространстве $(\Omega, B)$ задана мера $\mu$, где $B$ является $\sigma$-алгеброй, порожденной конечными цилиндрическими подмножествами $\Omega$. Построим состояние $\omega_{\mu}$ на $A$ следуюшим образом. Положим $\omega_{\mu}(x)=0$, если тензорный одночлен $x$ от матричных единиц $e_{i j}, i, j \in\{1,2\}$, содержит по крайней мере одну частичную изометрию. Если же $x \in \mathrm{C} A$, то положим $\omega_{\mu}(x)=\int_{\Omega} x d \mu$. Состояние, построенное таким образом, было введено в [9] и называлось диагональным.

Обозначим через $\omega_{0}$ диагональное состояние, порожденное экстремальной мерой $\mu_{0}$. Ясно, что состояние $\omega_{0}$ является экстремальным гиббсовским состоянием для квантованной модели Поттса, гамильтониан которой в силу (3) имеет следуюший вид:

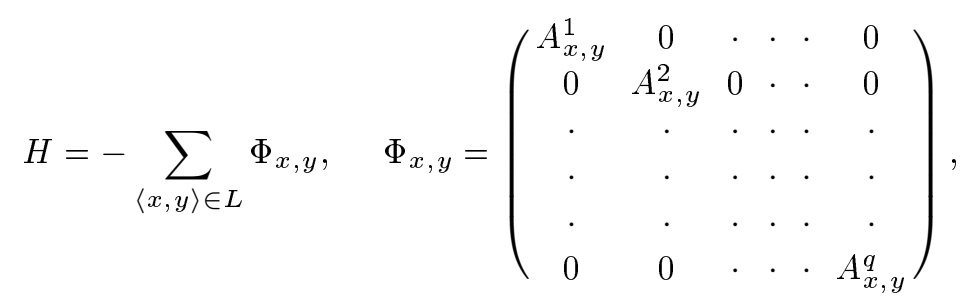

2 Теоретическая и математическая физика, т. 126, № 2, 2001 г. 
где

$$
A_{x, y}^{k}=\left(\ln p_{i j, k}^{\langle x, y\rangle}\right)_{i, j=1}^{q}
$$

- матрица $q \times q ; k=\overline{1, q} ;\left(p_{i j, k}^{\langle x, y\rangle}\right)$ - стохастическая матрица, которая определяется следуюшим образом:

если $x \in W_{2 n}, y \in W_{2 n+1},\langle x, y\rangle \in L, n \geqslant 0$, то

$$
\begin{gathered}
p_{k k, k}^{\langle x, y\rangle}=\frac{e^{\frac{J^{\prime}}{T}}}{\Xi_{1}}, \quad k=\overline{1, q}, \\
p_{i i, l}^{\langle x, y\rangle}=\frac{e^{-\frac{J^{\prime}}{(q-1) T}}}{\Xi_{1}}, \quad i, l=\overline{1, q}, \quad l \neq i, \\
p_{i j, l}^{\langle x, y\rangle}=0, \quad i, j, l=\overline{1, q}, \quad i \neq j, \\
\Xi_{1}=e^{\frac{J^{\prime}}{T}}+(q-1) e^{-\frac{J^{\prime}}{(q-1) T}}
\end{gathered}
$$

если $x \in W_{2 n-1}, y \in W_{2 n},\langle x, y\rangle \in L, n \geqslant 1$, то

$$
\begin{gathered}
p_{k k, k}^{\langle x, y\rangle}=\frac{e^{-\frac{J^{\prime}}{T}}}{\Xi_{2}}, \quad k=\overline{1, q}, \\
p_{i i, l}^{\langle x, y\rangle}=\frac{e^{\frac{J^{\prime}}{(q-1) T}}}{\Xi_{2}}, \quad i, l=\overline{1, q}, \quad l \neq i, \\
p_{i j, l}^{\langle x, y\rangle}=0, \quad i, j, l=\overline{1, q}, \quad i \neq j, \\
\Xi_{2}=e^{-\frac{J^{\prime}}{T}}+(q-1) e^{\frac{J^{\prime}}{(q-1) T}} .
\end{gathered}
$$

Обозначим $\mathcal{M}=\pi_{\omega_{0}}(A)$, где $\pi_{\omega_{0}}-$ ГНС-представление, ассоциированное с состоянием $\omega_{0}$ (см. определение 2.3 .18 в [6]). Из экстремальности $\omega_{0}$ вытекает, что $\mathcal{M}$ является фактором. Модулярная группа на $\mathcal{M}$ определяется следуюшим образом:

$$
\sigma_{t}^{\omega_{0}}(x)=\lim _{\Lambda \rightarrow \Gamma^{k}} \exp \{i t H(\Lambda)\} x \exp \{-i t H(\Lambda)\}, \quad x \in \mathcal{M}
$$

где

$$
H(\Lambda)=\sum_{\langle x, y\rangle \in \Lambda} \Phi_{x, y} .
$$

Сушествование последнего предела доказано в [10] (теорема 6.2.4). Обозначим через $\mathcal{M}^{\sigma}$ централизатор $\sigma^{\omega_{0}}$, определенный следующим образом:

$$
\mathcal{M}^{\sigma}=\left\{x \in \mathcal{M}: \sigma_{t}^{\omega_{0}}(x)=x \forall t \in \mathbb{R}\right\}
$$

Пользуясь экстремальностью состояния $\omega_{0}$, аналогично тому, как это делалось в [11], можно доказать следуюшую лемму. 
Лемма 1. Централизатор $\mathcal{M}^{\sigma}$ является фактором типа $\mathrm{II}_{1}$.

Теорема 2. Если выполняется условие (5), то алгебра фон Неймана $\mathcal{M}$, соответствующая неупорядоченной фазе неоднородной модели Поттса (4) на дереве Кәли, является фактором типа $\mathrm{III}_{1}$.

ДокАЗАТЕЛЬСтво. В силу леммы 1 имеем $Z\left(\mathcal{M}^{\sigma}\right)=\mathbb{C} 1$. Тогда из предложения 2.2.2 работы [7] вытекает $\Gamma\left(\sigma^{\omega_{0}}\right)=S p\left(\sigma^{\omega_{0}}\right)$, где $S p\left(\sigma^{\omega_{0}}\right)$ - спектр Арвесона.

Рассмотрим оператор

$$
H\left(V_{n}\right)=\sum_{\langle x, y\rangle \in L_{n}} \Phi_{x, y}
$$

Через $S p\left(H\left(V_{n}\right)\right)$ обозначим спектр оператора $H\left(V_{n}\right)$. Если положить

$$
\sigma_{t}^{\omega_{0}, n}(x)=\exp \left\{i t H\left(V_{n}\right)\right\} x \exp \left\{-i t H\left(V_{n}\right)\right\}, \quad x \in \mathcal{M},
$$

TO

$$
S p\left(\sigma^{\omega_{0}, n}\right)=S p\left(H\left(V_{n}\right)\right)-S p\left(H\left(V_{n}\right)\right)=\left\{\lambda-\mu: \lambda, \mu \in S p\left(H\left(V_{n}\right)\right)\right\} .
$$

Ясно, что $\ln p_{i j, k}^{\langle x, y\rangle} \in S p\left(H\left(V_{n}\right)\right) \forall i, j, k \in \overline{1, q}, x, y \in V_{n}$. Из (6) следует, что $S p\left(\sigma^{\omega_{0}, n}\right)$ порождается элементами вида

$$
\ln \left\{\frac{p_{i j, k}^{\langle x, y\rangle}}{p_{m l, k^{\prime}}^{\left\langle x^{\prime}, y^{\prime}\right\rangle}}\right\}, \quad i, j, k, l, m, k^{\prime} \in \overline{1, q}, \quad\langle x, y\rangle,\left\langle x^{\prime}, y^{\prime}\right\rangle \in L_{n} .
$$

Очевидно, что имеет место равенство

$$
\frac{p_{i i, k}^{\langle x, y\rangle}}{p_{j j, l}^{\langle x, y\rangle}}=\lambda^{\varepsilon_{j j, l}^{i i, k}}(x, y)
$$

где $\varepsilon_{j j, l}^{i i, k} \in\{-1,0,1\}$,

$$
\lambda(x, y)=\left\{\begin{array}{ll}
e^{\frac{J^{\prime} q}{(q-1) T}}, \quad x \in W_{2 n}, \quad y \in W_{2 n+1}, \quad n \geqslant 0, \\
e^{-\frac{J^{\prime} q}{(q-1) T}}, \quad x \in W_{2 n-1}, \quad y \in W_{2 n}, \quad n \geqslant 1,
\end{array} \quad\langle x, y\rangle \in L .\right.
$$

Обозначим $\lambda=e^{\frac{J^{\prime} q}{(q-1) T}}$.

ЛЕмма 2. Ни при каких значениях $m \in \mathbb{Z} u T>0$ равенство

$$
\left(\frac{e^{\frac{J^{\prime}}{T}}}{\Xi_{1}}\right) /\left(\frac{e^{-\frac{J^{\prime}}{T}}}{\Xi_{2}}\right)=\lambda^{m}
$$

не выполняется. 
ДОКАЗАТЕЛЬСТво. Из (7) полУчИм

$$
\frac{1+(q-1) \lambda}{1+(q-1) \lambda^{-1}}=\lambda^{m}
$$

Из неравенств $1+(q-1) \lambda>1+(q-1) \lambda^{-1}, \lambda>1$ вытекает, что $m \geqslant 1$. Тогда последнее равенство имеет следуюший вид:

$$
\frac{1+(q-1) \lambda}{\lambda+(q-1)}=\lambda^{m-1}
$$

Отсюда получим следуюшее уравнение:

$$
\lambda^{m}-1+(q-1) \lambda\left(\lambda^{m-2}-1\right)=0
$$

Предположим, что $m>2$, тогда имеем

$$
\lambda^{m-1}+q \lambda^{m-2}+q \lambda^{m-3}+\cdots+q \lambda+1=0 .
$$

Это равенство не выполняется ни при каких значениях $m>2$ и $T>0$, так как $\lambda>1$.

Пусть теперь $m \in\{1,2\}$. Тогда непосредственной проверкой можно убедиться, что равенство (8) также не выполняется. Лемма доказана.

Из доказанной леммы вытекает, что не существует целых чисел $m\left(i, j, k, l, q, k^{\prime}, x, y\right.$, $\left.x^{\prime}, y^{\prime}\right) \in \mathbb{Z}$ таких, что

$$
\frac{p_{i j, k}^{\langle x, y\rangle}}{p_{q l, k^{\prime}}^{\left\langle x^{\prime}, y^{\prime}\right\rangle}}=\lambda^{m\left(i, j, k, l, q, k^{\prime}, x, y, x^{\prime}, y^{\prime}\right)},
$$

где $i, j, k, l, q, k^{\prime} \in \overline{1, q},\langle x, y\rangle,\left\langle x^{\prime}, y^{\prime}\right\rangle \in L$. Так как $S p\left(\sigma^{\omega_{0}}\right)$ состоит из предельных точек множества $\bigcup_{n} S p\left(\sigma^{\omega_{0}, n}\right)$ (см. [12]), то из этого факта и соотношения (9) следует, что $\Gamma\left(\sigma^{\omega_{0}}\right)=\mathbb{R}$. Последнее означает, что фактор $\mathcal{M}$ имеет тип III ${ }_{1}$. Теорема доказана.

Заметим, что задача определения типа алгебр фон Неймана, ассоциированных через ГНС-конструкцию с экстремальными фазами, может быть изучена также для моделей, рассмотренных в [13]-[15].

Благодарности. Авторы выражают глубокую признательность проф. Н. Н. Ганиходжаеву за внимание к работе. 


\section{Список литературы}

[1] H. Araki, E. J. Woods. Publ. R. I. M. S. (Kyoto Univ.). 1968. № 3. P. 51-130.

[2] I. F. Wilde. J. Funct. Anal. 1974. V. 15. P. 12-21.

[3] R. Powers. Ann. Math. 1967. № 81. P. 138-171.

[4] Ф. М. Мухамедов. ТМФ. 2000. Т. 123. № 1. С. 83-88.

[5] Ф. М. Мухамедов, У. А. Розиков. ТМФ. 2000. Т. 124. № 3. С. 410-418.

[6] У. Браттели, Д. Робинсон. Операторные алгебры и квантовая статистическая механика. М.: Мир, 1982.

[7] A. Connes. Ann. Ec. Norm. Sup. 1973. V. 6. P. 133-252.

[8] S. Stratila. Modular theory in operator algebras. Bucuresti: Abacus Press, 1981.

[9] А. Г. Шухов. Функц. анализ и его прилож. 1980. Т. 14. № 2. С. 95-96.

[10] O. Bratteli, D. Robinson. Operator algebras and Quantum Statistical Mechanics II. Berlin: Springer, 1981.

[11] N. N. Ganikhodjaev, F. M. Mukhamedov. Methods of Funct. Anal. and Topol. 1998. V. 4. № 3. P. 33-38.

[12] M. Takesaki. Reg. Conf. Ser. Math. 1983. V. 51. P. 1-107.

[13] Н. Н. Ганиходжсаев. ДАН РУз. 1992. № 6-7. С. 4-7.

[14] Н. Н. Ганиходжсаев, У. А. Розиков. ТМФ. 1997. Т. 111. № 1. С. 109-117.

[15] Н. Н. Ганиходжсаев, У. А. Розиков. Матем. сб. 1999. Т. 190. № 2. С. 31-42.

Поступила в редакцию 21.VI.2000 г., после доработки 8.Х.2000 г. 